\title{
Prolonged Coagulopathy Following Rattlesnake Envenomation Responsive to Antivenom: A Case Report
}

\author{
Megan Z Roberts ${ }^{1 *}$, Brian P McKinzie ${ }^{1}$, Stuart M Leon ${ }^{2}$, Stephen A Fann ${ }^{2}$ and Nicole M Bohm \\ ${ }^{1}$ Department of Pharmacy Services, Medical University of South Carolina, USA \\ ${ }^{2}$ Department of Surgery, Medical University of South Carolina, USA \\ ${ }^{3}$ Department of Clinical Pharmacy and Outcomes Sciences, South Carolina College of Pharmacy, USA
}

*Corresponding author: Megan Z Roberts, Department of Pharmacy Services, Medical University of South Carolina, Charleston, USA, E-mail: zeek@musc.edu

\begin{abstract}
Introduction: Venom-specific immunoglobulin G fragments are used for the management of patients with North American crotalid envenomation. In this case, ongoing coagulopathy and clinically significant sequelae required a protracted course of antivenom therapy in a patient experiencing severe rattlesnake envenomation.

Case: A 51-year-old male presented with breathing difficulties, hypotension, and coagulopathy following envenomation of his right forearm by an Eastern Diamondback rattlesnake (C. adamanteus). He required intubation, blood products, and an initial 10-vial dose of antivenom ( $\mathrm{CroFab}^{\circledR}$ ). On hospital day 2, the patient was considered to have completed antivenom therapy after receiving 34 vials. However, due to recurrent coagulopathies the patient ultimately received 45 antivenom vials, with a final dose 12 days after envenomation. His 30-day hospitalization was complicated by kidney injury requiring continuous renal replacement therapy, pneumonia, and the need for vasopressor support.
\end{abstract}

Conclusion: Antivenom may be effective for treating patients with delayed, recurrent coagulopathy subsequent to a large envenomation and circulatory collapse.

\section{Keywords}

Antivenom, Rattlesnake envenomation, Recurrence phenomenon, Coagulopathy, Circulatory collapse

\section{Introduction}

In North America, the majority of venomous snake bites are from the Viperidae subfamily Crotalinae, which includes rattlesnakes. The extent of injury due to crotalid envenomation varies greatly depending on charac- teristics of the snake and victim [1]. The cytotoxins and proteolytic enzymes of rattlesnake venom can result in local erythema, ecchymosis, compartment syndrome, neurologic deficits, renal failure, and respiratory compromise. Hematologic abnormalities associated with rattlesnake envenomation include coagulopathy, hypofibrinogenemia, and thrombocytopenia due to fibrin degradation and platelet destruction [1,2].

$\mathrm{CroFab}^{\circledR}$ is a venom-specific Fab fragment of immunoglobulin $G$ which binds and neutralizes toxins $[3,4]$. This mainstay of therapy for crotalid snakebites is used for patients experiencing progressive local, hematologic, and systemic effects of envenomation [3]. Typically, 1 or 2 doses of 4 to 6 antivenom (CroFab ${ }^{\circledR}$ ) vials are given to achieve initial control [4]. Three maintenance doses may be utilized to prevent recurrence of symptoms $[4,5]$ and patients can be discharged 24 hours after initial control [4].

\section{Case Report}

A 51-year-old man presented to a local community hospital within 2 hours of a bite on the right forearm from his 6-foot-long pet Eastern Diamondback rattlesnake (C. adamanteus). He received 10 vials of antivenom and 4 units of Fresh Frozen Plasma (FFP) and was intubated prior to being transferred to a tertiary care academic medical center. His medical history included anxiety and hypertension; his only home medication was alprazolam $0.5 \mathrm{mg}$. Upon arrival at our hospital 2.5 hours after envenomation, 


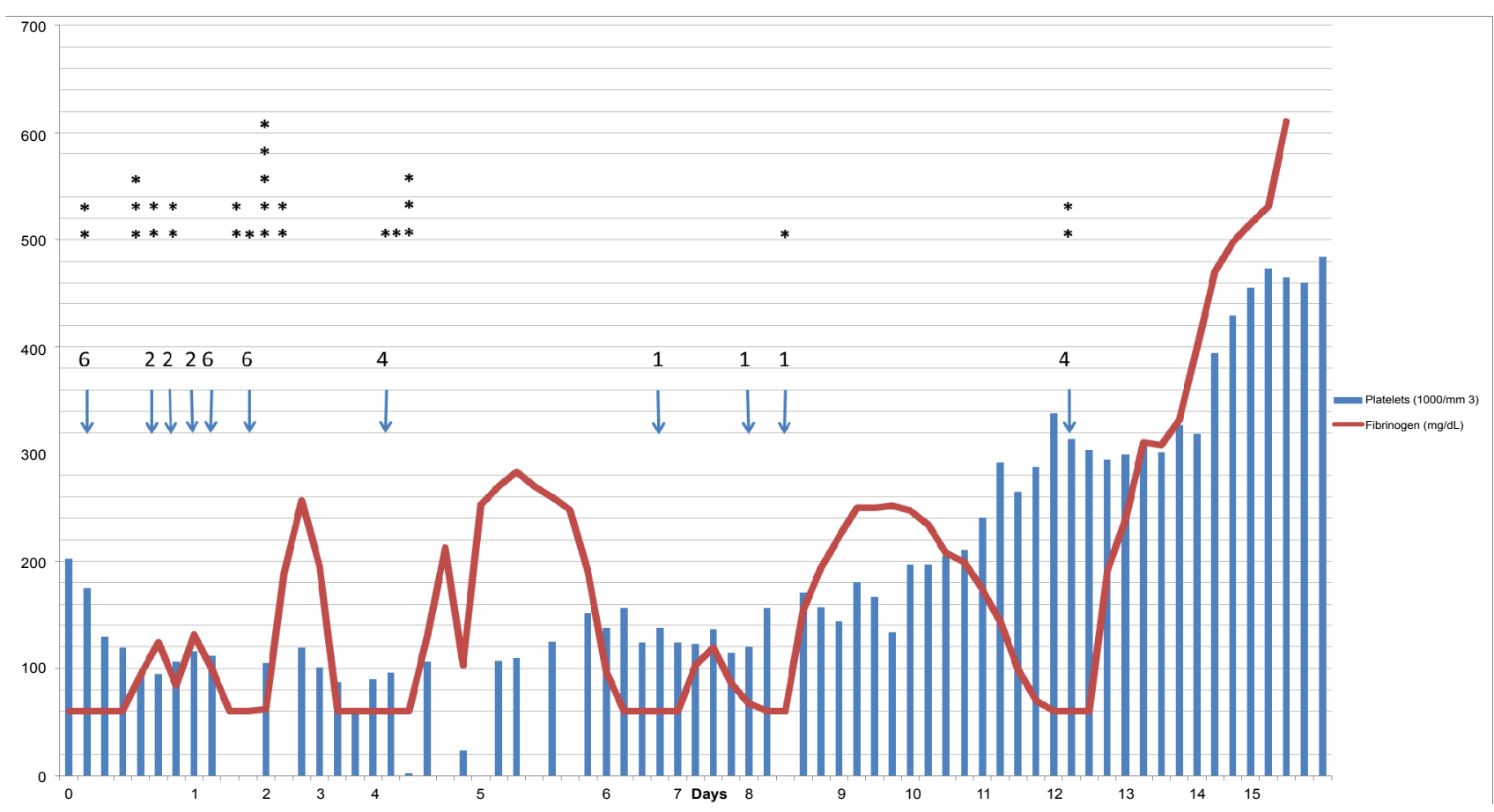

Figure 1: Evolution and resolution of $C$. adamanteus coagulopathy.

* indicates timing and number of cryoprecipitate units administered and $\downarrow$ indicates timing and number of antivenom vials administered.

the physical exam findings included blood oozing from the urethral meatus around the foley catheter and from four puncture wounds on his forearm. Laboratory values were consistent with coagulopathy from envenomation (Figure 1). Throughout hospital day one, the patient received antivenom, FFP, and cryoprecipitate due to persistent coagulopathies and development of a gastrointestinal bleed with melenic stool. On hospital day 2, coagulation parameters normalized and clinical bleeding ceased resulting in the discontinuation of antivenom and management of hypofibroginemia with cryoprecipitate. However, later that day, coagulation parameters worsened and antivenom was reinitiated along with FFP and additional cryoprecipitate. The coagulopathy resolved on hospital day 14 after the patient had received a cumulative 45 vials of antivenom (CroFab $\left.{ }^{\circledR}\right), 21$ units of FFP, 7 units of Packed Red Blood Cells (PRBC), and 27 units of cryoprecipitate. The final 4 vials of antivenom were administered under the guidance of a poison control expert on hospital day 12 due to concern for worsening coagulopathy in the setting of hypotension and bloody output from the nasogastric tube.

The patient's hospital course was complicated by methicillin-susceptible Staphylococcus aureus pneumonia, hypotension necessitating vasopressor therapy, and acute kidney injury requiring continuous veno-venous hemodiafiltration from hospital day 10 through 16 followed by intermittent hemodialysis. On hospital day 17, after completion of therapy for pneumonia, a tracheostomy was performed. Decannulation occurred on hospital day 28. Eventually the patient's renal recovery was sufficient to discontinue hemodialysis, and he was discharged home on hospital day 30.

\section{Discussion}

Antivenom can minimize systemic effects, arrest spread of local tissue effects, and reduce envenomation-related coagulopathies with improvement in fibrinogen and platelet levels within 1 hour of administration $[4,6]$. The recommended starting dose for antivenom $\left(\mathrm{CroFab}^{\circledR}\right)$ is 4 to 12 vials depending on clinical judgment and envenomation severity. This should be repeated until initial control is achieved and followed by 3 maintenance doses of 2 vials every 6 hours $[4,5]$. Additional 2-vial doses may be administered based on the patient's clinical course [5]. Failure to achieve initial control (clinical stability, no progression in swelling, and improvement in coagulopathies) after 2 doses of antivenom is considered uncommon by expert clinical consensus [4]. Overall $82.2 \%$ of patients achieved initial control in a retrospective study of 247 crotalid envenomations [7] compared to $57 \%$ of severely envenomated patients [8].

In this case, initial antivenom dosing was consistent with current dosing recommendations. However, initial control was not achieved after 2 doses, and the patient required repeated doses over a 12-day span, which is among the longest durations of antivenom therapy described. Neurologic deficits and thrombocytopenia prior to administration of antivenom are independently associated with failure to achieve initial control, both of which occurred in this patient [7]. The recurrence phenomenon and unique clinical aspects of this case may have also contributed to the protracted course. Recurrence of coagulopathy following antivenom therapy occurs in $45 \%$ to $53 \%$ of patients up to 14 days after crotalid envenomation $[9,10]$. Mechanisms to explain the phenomenon include (1) Pharmacokinet- 
ic mismatch of the shorter half-life antivenom compared with venom components [11], (2) A depot of unneutralized venom, (3) Separation of venom complexed to antivenom in systemic circulation after neutralization, (4) Presence of venom components with late onset effects, and (5) Immune-Mediated response to antivenom [12]. The patient's receipt of FFP, cryoprecipitate, and PRBC may have produced a transient improvement in coagulation parameters masking the need for more aggressive antivenom administration throughout the patient's course [4].

In addition, the effect of circulatory collapse leading to the reduced ability of blood flow to distribute antivenom to unneutralized venom depot or the antivenom itself to be released from the depot site has not previously been studied but may have contributed to this patient's prolonged envenomation-related toxicities. Recent in vitro studies propose the possibility of inactivating a venom depot using regional administration of carbon monoxide and iron preparations as additional therapy to antivenom. These preclinical studies demonstrate the ability of iron and carbon monoxide to attenuate venom mediated fibrinogen catalysis via modification of fibrinogen rendering it less susceptible to venom proteases, including those expressed by Eastern diamondback rattlesnakes $[13,14]$. While further studies are warranted future cases such as ours may benefit from complimentary, regional carbon monoxide and iron therapy if available.

The requirement for 45 vials of antivenom is exceeded by only two other crotalid envenomation reports. One required 46 vials; however the clinical details of the case are not described [7]. The other describes a 50-year-old man also bitten by a 6-foot Eastern Diamondback rattlesnake requiring 51 total vials of antivenom with the last 6 vials given as continuous intravenous infusion over 36 hours to dissipate the recurrent coagulopathy on day 8 . In this case no indication of circulatory collapse or receipt of blood products was described [15]. The large size of snake in the aforementioned case and our case likely impacted the dose required. Increasing rattlesnake size is associated with a higher snakebite severity score, which correlates with the need for more antivenom and longer hospitalization [16]. An important implication regarding the doses used in these cases was the lack of serum sickness. The risk of serum sickness may relate to antivenom dose and the associated protein load [4]. One report of non-serious serum sickness occurred in a patient receiving 34 vials [8], but the low incidence overall precludes clearly correlating incidence to dosing. Our case provides evidence for safe administration of 45 cumulative vials in this patient, but clinicians should monitor for the adverse event.

Our case also provides novel evidence indicating the success and safety of regional citrate anticoagulation in maintaining circuit patency in patient's undergoing Continuous Renal Replacement Therapy (CRRT) while experiencing envenomation associated coagulopathies. Addition of exogenous citrate has been shown in vitro to inhibit venom protease activity via chelation with zinc in zinc-dependent enzymes, however to a far less extent with the Crotalinae subfamily compared to other vipers, such as the Bothrops genus (7.8 vs. 75\%) [17]. Further studies and reports could further assess benefits of citrate anticoagulation in patients requiring CRRT after envenomation with various viper species.

\section{Conclusion}

This case report illustrates ongoing coagulopathy and concern for recurrent gastrointestinal bleeding in a victim of severe rattlesnake envenomation, which responded to treatment with antivenom 12 days post-envenomation. The prolonged course and large number of antivenom vials are unique. It is possible that circulatory collapse and rattlesnake size contributed to the recurrence phenomenon. Further studies evaluating potential complementary therapy including carbon monoxide and iron as well as the use of citrate anticoagulation with CRRT may provide exciting therapeutic advancements for future similar cases.

\section{Acknowledgements}

None to disclose.

\section{Disclosure Statement}

The authors report no conflicts of interest. The authors alone are responsible for the content and writing of this article.

\section{References}

1. Spiller HA, Bosse GM, Ryan ML (2010) Use of antivenom for snakebites reported to United States poison centers. Am J Emerg Med 28: 780-785.

2. Anz AW, Schweppe M, Halvorson J, Bushnell B, Sternberg $M$, et al. (2010) Management of venomous snakebite injury to the extremities. J Am Acad Orthop Surg 18: 749-759.

3. Tanen D, Ruha A, Graeme K, Curry S (2001) Epidemiology and hospital course of rattlesnake envenomations cared for at a tertiary referral center in Central Arizona. Acad Emerg Med 8: 177-182.

4. Lavonas EJ, Ruha AM, Banner W, Bebarta V, Bernstein $\mathrm{JN}$, et al. (2011) Unified treatment algorithm for the management of crotaline snakebite in the United States: results of an evidence-informed consensus workshop. BMC Emerg Med 11: 2

5. (2016) $\mathrm{CroFab}^{\circledR}$ - ovine crotalide venoms immune fab injection. BTG International Inc, West Conshohocken, PA.

6. Dart RC, Seifert SA, Boyer LV, Clark RF, Hall E, et al. (2001) A randomized multicenter trial of crotalinae polyvalent immune Fab (ovine) antivenom for the treatment for crotaline snakebite in the United States. Arch Intern Med 161: 2030-2036.

7. Yin S, Kokko J, Lavonas E, Mlynarchek S, Bogdan G, et al. (2011) Factors associated with difficult achieving initial control with crotalide polyvalent immune fab antivenom in snakebite patients. Acad Emerg Med 18: 46-52. 
8. Lavonas EJ, Kokko J, Schaeffer TH, Mlynarchek SL, Bogdan GM, et al. (2011) Short-term outcomes after Fab antivenom therapy for severe crotaline snakebite. Ann Emerg Med 57: 128-137.

9. Boyer LV, Seifert SA, Clark RF, McNally JT, Williams SR, et al. (1999) Recurrent and persistent coagulopathy following pit viper envenomation. Arch Intern Med 159: 706-710.

10. Bogdan GM, Dart RC, Falbo SC, McNally J, Spaite D (2000) Recurrent coagulopathy after antivenom treatment of crotalid snakebite. South Med J 93: 562-566.

11. Seifert SA, Boyer LV (2001) Recurrence phenomena after immunoglobulin therapy for snake envenomations: Part 1. Pharmacokinetics and pharmacodynamics of immunoglobulin antivenoms and related antibodies. Ann Emerg Med 37: 189-195.

12. Boyer LV, SA Seifert, Cain JS (2001) Recurrence phenomena after immunoglobulin therapy for snake envenomations: part 2. Guidelines for clinical management with crotaline fab antivenom. Ann Emerg Med 37: 196-210.
13. Nielsen VG (2016) Iron and carbon monoxide prevent degradation of plasmatic coagulation by thrombin-like activity in rattlesnake venom. Hum Exp Toxicol 35: 1116-1122.

14. Nielsen VG, Bazzell CM (2017) Carbon monoxide releasing molecule-2 inhibition of snake venom thrombin-like activity: novel biochemical "brake"? J Thromb Thrombolysis 43: 203-208.

15. Hwang CW, Flach Fe (2015) Recurrent coagulopathy after rattlesnake bite requiring continuous intravenous dosing of antivenom. Case Rep Emerg Med 2015: 719302.

16. Janes DN Jr, Bush SP, Kolluru GR (2010) Large snake size suggests increased snakebite severity in patients bitten by rattlesnakes in Southern California. Wilderness Environ Med 21: 120-126.

17. Odell GV, Ferry PC, Vick LM, Fenton AW, Decker LS, et al. (1998) Citrate inhibition of snake venom proteases. Toxicon 36: 1801-1806. 